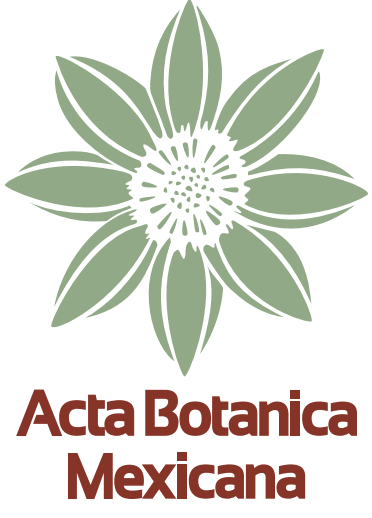

\author{
Gustavo Enrique Mendoza-Arroyo' (D), Alejandro Morón-Ríos,4 (D), Mario González-Espinosa² (D), José Armando Alayón- \\ Gamboa' (D), Pedro Antonio Macario-Mendoza³ (D)
}

\section{La supervivencia y desarrollo de plántulas de Brosimum alicastrum (Moraceae) y Psidium sartorianum (Myrtaceae) difieren en condiciones de inundación}

\title{
Resumen:
}

Antecedentes y Objetivos: En los bosques tropicales la inundación estacional puede actuar como filtro ambiental afectando la supervivencia, el establecimiento y el crecimiento de las plántulas de especies arbóreas e influir en la estructura de las comunidades. Se simularon condiciones de inundación y sequía con el objetivo de evaluar su efecto como filtro ambiental sobre la supervivencia, crecimiento (longitud del tallo y número de hojas) y biomasa acumulada en plántulas de Brosimum alicastrum y Psidium sartorianum de la selva de Campeche, México.

Métodos: Se estableció un experimento en condiciones de vivero con un diseño completamente al azar, con tres tratamientos (sequía, riego regular e inundación). Para cada tratamiento se utilizaron 45 plántulas de cada especie. Se evaluaron la supervivencia, altura y número de hojas de las plántulas al inicio del experimento y a intervalos de 30 días hasta su conclusión a los 120 días. Al finalizar, se cosecharon las plántulas y se midió la longitud de las raíces y la biomasa acumulada.

Resultados clave: La inundación disminuyó la supervivencia de plántulas de B. alicastrum y no la de $P$. sartorianum, limitó la longitud del tallo, el número de hojas y la biomasa acumulada de ambas especies, pero no la biomasa de raíces de $P$. sartorianum. La supervivencia de las plántulas expuestas a sequía y riego regular no cambió y aumentó el crecimiento y biomasa acumulada.

Conclusiones: Las respuestas de las especies contribuyeron a entender el papel del filtro ambiental en el establecimiento de las plántulas de especies arbóreas en los bajos de Calakmul, Campeche. La inundación estacional actúa como un filtro ambiental, elimina las plántulas de $B$. alicastrum en los bajos, mientras que esta especie predomina en áreas con suelos bien drenados como los lomeríos adyacentes a la zona inundada. Psidium sartorianum se adaptó a las condiciones de sequía e inundación, como se observa en su hábitat natural.

Palabras clave: cambio climático, filtro ambiental, selvas inundables, suelos inundados.

\section{Abstract:}

Background and Aims: Seasonal flooding in tropical forests may function as an environmental filter that affects the survival, establishment and growth of tree seedlings, with consequences for the assembly of communities. Seasonal flood and drought conditions were simulated with the objective to evaluate their effect on the survival, growth (stem length and number of leaves), as well as biomass accumulation in Brosimum alicastrum and Psidium sartorianum seedlings from the tropical forest of Campeche, Mexico.

Methods: A nursery experiment was established with a completely random design with three treatments (flooding, regular irrigation and drought). For each treatment 45 seedlings were used for each species. The survival, height and number of leaves of the seedlings were measured at the beginning of the experiment and at 30 days intervals until the experiment ended at 120 days. The seedlings were then harvested and the length of the roots and the biomass accumulation were measured.

Key results: The flood decreased the survival of $B$. alicastrum and did not change the survival of $P$. sartorianum. Likewise, it limited the length of the stem, the number of leaves and the biomass accumulated in both species, except for the biomass in roots of $P$. sartorianum. The survival of the seedlings exposed to drought and regular irrigation were not altered and showed increases in growth and accumulated biomass.

Conclusions: The responses of the species contributed to understand the role of the environmental filter in the establishment of arboreal species seedlings in the lowlands of Calakmul, Campeche. Seasonal flooding in the lowlands acts as an environmental filter because it eliminates B. alicastrum seedlings, while it predominates in areas with well-drained soils, such as the hills adjacent to the low floodplains. Psidium sartorianum was well adapted to the conditions of drought and flood, as observed in its natural habitat.

Key words: climate change, environmental filter, flooded soils, lowland forest.

${ }^{1}$ El Colegio de la Frontera Sur (ECOSUR), Departamento de Conservación de la Biodiversidad, Av. Rancho, Polígono 2A, Ciudad Industrial de Lerma, 24500 San Francisco de Campeche, Campeche, México.

${ }^{2}$ El Colegio de la Frontera Sur (ECOSUR), Departamento de Conservación de la Biodiversidad, Carretera Panamericana y Periférico Sur s.n., 29290 San Cristóbal de Las Casas, Chiapas, México.

${ }^{3}$ El Colegio de la Frontera Sur (ECOSUR), Departamento de Agricultura, Sociedad y Ambiente, Avenida Centenario km 5.5, Colonia Pacto Obrero Campesino, 77014 Chetumal, Quintana Roo, México.

${ }^{4}$ Autor para la correspondencia: amoron@ecosur.mx, moriosa08@gmail.com
Recibido: 12 de abril de 2019 Revisado: 10 de junio de 2019.

Aceptado por Moisés Méndez Toribio: 27 de septiembre de 2019.

Publicado Primero en línea: 3 de diciembre de 2019.

Publicado: Acta Botanica Mexicana 127 (2020).
Citar como:

Mendoza-Arroyo, G. E., A. Morón-Ríos, M. GonzálezEspinosa, J. A. Alayón-Gamboa y P. A. Macario-Mendoza. 2019(2020). La supervivencia y desarrollo de plántulas de Brosimum alicastrum (Moraceae) y Psidium sartorianum (Myrtaceae) difieren en condiciones de inundación. Acta Botanica Mexicana 127: e1548. DOI: 10.21829/abm127.2020.1548
Este es un artículo de acceso abierto bajo la licencia Creative Commons 4.0 Atribución-No BY-NC 4.0 International). 


\section{Introducción}

En los bosques tropicales húmedos, las inundaciones constituyen un filtro ambiental que influye en el establecimiento de plántulas de especies arbóreas (Meli et al., 2015; Conserva et al., 2018); este filtro actúa a pesar de la existencia de un proceso de dispersión de semillas desde los bosques adyacentes localizados en áreas con suelos bien drenados hacia el interior de los fragmentos estacionalmente inundables (Pitman et al., 2014).

La hipótesis del filtro ambiental postula que las condiciones abióticas seleccionan, del conjunto regional de especies, a aquellas con atributos similares (Keddy, 1992). A medida que dichas condiciones son más severas, es decir, cuando la duración y el nivel de inundación aumentan, pueden ocurrir extinciones locales de especies incluso dentro del mismo género, lo que conduce a un agrupamiento filogenético (Webb, 2000). Un resultado de la operación del filtro ambiental es que la riqueza local de especies en los hábitats más estresantes puede ser sólo una parte de la existente en los fragmentos forestales circundantes (Kraft et al., 2015; Cadotte y Tucker, 2017).

Cuando la inundación estacional actúa como un filtro ambiental severo puede potenciar los procesos de especialización y diversificación que ocurren dentro de las zonas inundables (Wittmann et al., 2013; Luize et al., 2018) e incluso puede propiciar la dominancia de algunas especies (López y Kursar, 2007).

Para entender el efecto individual de un filtro ambiental (por ej. inundaciones o sequía), Kraft et al. (2015) señalan que se requiere evaluar la tolerancia de las especies a factores abióticos en ausencia de otras especies competidoras. Esto permite comprender con mayor claridad el efecto de las condiciones abióticas que influyen en el desarrollo, establecimiento y reclutamiento de plántulas (Meli et al., 2015; Araújo da Paz et al., 2017; Cadotte y Tucker, 2017; Conserva et al., 2018).

La inundación estacional actúa sobre las plántulas de especies arbóreas y provoca limitaciones en su crecimiento (López y Kursar, 2003; Almeida et al., 2018), pérdida de hojas (Parolin, 2001; Schöngart et al., 2002; Hawes y Peres, 2016), disminución de la acumulación de biomasa total (Schranm et al., 2005) y también afecta negativamente la longitud de las raíces y la proporción raíz:tallo (López y
Kursar, 1999). Como respuesta, algunas especies de plantas han desarrollado mecanismos, como la producción de raíces aéreas (Wittmann y Parolin, 2005), que permiten su supervivencia (De Simone et al., 2003). Estos ajustes morfo-fisiológicos brindan a las especies la posibilidad de sobrevivir en áreas temporalmente inundables (Soares et al., 2018). Los patrones y mecanismos del establecimiento de las plántulas de árboles ayudan a explicar la distribución de los individuos adultos en gradientes de inundación (Parolin, 2002), con consecuencias en la composición florística y estructura de la comunidad vegetal (Pitman et al., 2014; Assis et al., 2015; Myster, 2015; Araújo da Paz et al., 2017).

En la región sur de la Península de Yucatán, México, los fragmentos de selva inundables tienen un menor número de especies en comparación con las selvas medianas subcaducifolias circundantes y localizadas en áreas con suelos bien drenados (Duch-Gary, 1989; Martínez y Galindo-Leal, 2002; Albor-Pinto et al., 2017). El objetivo de este estudio fue evaluar el efecto del anegamiento y sequía sobre la supervivencia, el crecimiento (longitud del tallo y número de hojas) y la acumulación de biomasa en plántulas de Brosimum alicastrum Sw., una especie típica de la selva mediana subcaducifolia (Martínez y Galindo-Leal, 2002; Pennington y Sarukhán, 2005; Bonilla-Moheno y Holl, 2010) que se desarrolla en condiciones no inundables y de Psidium sartorianum (O. Berg) Nied., una especie característica de la selva baja estacionalmente inundable (Pennington y Sarukhán, 2005).

\section{Materiales y Métodos}

\section{Selección y características de las especies}

En el sur de Campeche, México, Brosimum alicastrum es abundante en los lomeríos rocosos y bien drenados en donde se desarrollan rodales de selva mediana subcaducifolia, mientras que Psidium sartorianum es abundante en las depresiones o bajos inundables conformados por rodales de selva baja subperennifolia o selva baja inundable (Miranda y Hernández X., 1963; Vega-López et al., 2003; Pennington y Sarukhán, 2005). Ambas unidades del paisaje están separadas por distancias menores a 250 metros y pueden ser claramente identificadas y delimitadas por sus atributos topográficos, geomorfológicos y edáficos (Duch-Gary, 1989; Beach et al., 2008, 2016; Dunning et al., 2016). La 
escasa distancia que separa dichas unidades del paisaje posibilita la dispersión de semillas desde los bajos hacia los lomeríos circundantes y viceversa; en consecuencia, brinda la posibilidad de que las especies se establezcan en el hábitat vecino o que su establecimiento pueda ser limitado por la acción de un filtro ambiental. Por tal motivo, elegimos Brosimum alicastrum y Psidium sartorianum por encontrarse en ambientes con condiciones edáficas y topográficas contrastantes (Beach et al., 2006, 2008, 2018).

Brosimum alicastrum (Moraceae) (ramón) es una especie dominante en las selvas altas perennifolias, medianas subperennifolias y medianas subcaducifolias (Pennington y Sarukhán, 2005); se desarrolla en suelos bien drenados, sobre calizas con alta pedregosidad (VegaLópez et al., 2003). Es una especie arbórea de hasta 40 $\mathrm{m}$ de altura, diámetro a la altura del pecho de hasta 1.5 $\mathrm{m}$, tronco recto, contrafuertes grandes y bien formados, ramas ascendentes, luego colgantes, y copa piramidal y densa (Pennington y Sarukhán, 2005); la producción de sus frutos abarca tres periodos al año, durante los meses de febrero-marzo, mayo-agosto y noviembre-diciembre (FLORA DIGITAL, 2019).

Psidium sartorianum (Myrtaceae) (guayabillo, pichiche) es una especie que se desarrolla en suelos con alto contenido de arcillas, drenaje deficiente y sujetos a inundaciones estacionales (Duch-Gary, 1989; Martínez y Galindo-Leal, 2002; Palacio-Aponte et al., 2002). Se distribuye en selvas medianas subperennifolias, selvas bajas caducifolias (Pennington y Sarukhán, 2005) y selvas bajas inundables (FLORA DIGITAL, 2019). Es una especie arbórea cuya altura fluctúa entre 10 y $15 \mathrm{~m}$ de altura, tronco recto, ramas ascendentes y gruesas (Sánchez, 1990); la producción de sus frutos abarca los meses de junio a diciembre (FLORA DIGITAL, 2019).

\section{Recolecta de material}

Al final de la estación lluviosa (2 de noviembre de 2017) se colectaron 1200 plántulas de cada especie, provenientes de diversos árboles padres, sin daño aparente por herbivoría en las hojas no cotiledonarías. Las plántulas de $B$. alicastrum $(15.66 \pm 0.37 \mathrm{~cm}$ de altura) se colectaron en sitios de selva mediana subcaducifolia (no inundable) localizados en las coordenadas $18^{\circ} 33^{\prime} 34.88^{\prime \prime}$ de latitud
N y $89^{\circ} 53^{\prime} 39.00^{\prime \prime}$ de longitud O. Para el caso de las plántulas de $P$. sartorianum $(14.40 \pm 0.40 \mathrm{~cm}$ de altura) estas provinieron de un sitio con selva baja subperennifolia (inundable temporalmente), ubicado en las coordenadas $18^{\circ} 32^{\prime} 59.49^{\prime \prime}$ de latitud N y $89^{\circ} 56^{\prime} 24.50^{\prime \prime}$ de longitud $\mathrm{O}$. Ambos sitios de recolecta se localizan en la Península de Yucatán en las cercanías de la localidad de Conhuas, municipio Calakmul, Campeche, México.

\section{Establecimiento en vivero forestal}

Las plántulas recolectadas se transportaron al vivero forestal del Instituto Tecnológico de Chiná, Campeche, México. Las raíces de las plántulas se limpiaron con agua corriente e inmediatamente se plantaron en macetas de plástico de $800 \mathrm{ml}$ de capacidad, con $600 \mathrm{ml}$ de sustrato de agrolita comercial (Agrolita-Perlita ${ }^{\circledR}$ ).

Todas las plántulas se sometieron a un período de aclimatación de 30 días en el vivero forestal de tipo abierto dotado con malla-sombra de $50 \%$. El riego se efectuó cada $48 \mathrm{hrs}$ con agua embotellada. El agua utilizada presentó un pH neutro (7.0), conductividad eléctrica de 5-10 $\mathrm{mS} / \mathrm{cm}$, ambos parámetros se evaluaron con un conductímetro (Consort, Turnhout, Bélgica). Durante el período experimental se registró dentro del vivero la humedad relativa (promedio 78\%, mínima 70\%, máxima 82\%), la temperatura mínima (promedio $19{ }^{\circ} \mathrm{C}$, mínima $15{ }^{\circ} \mathrm{C}$, máxima $24{ }^{\circ} \mathrm{C}$ ) y la temperatura máxima (promedio $31^{\circ} \mathrm{C}$, mínima $22{ }^{\circ} \mathrm{C}$, máxima $37^{\circ} \mathrm{C}$ ), utilizando un equipo multi-parámetro digital (Traceable, Texas, EUA).

\section{Diseño experimental}

El experimento se basó en un diseño completamente al azar y tuvo una duración de 120 días ( 8 de diciembre de 2017-8 de abril de 2018). El criterio para finalizar el período experimental fue que cualquiera de las dos especies presentara más de $90 \%$ de mortalidad, independientemente del tratamiento al que estuvieran sometidas.

Después del período de aclimatación se eligieron aleatoriamente 135 plántulas de cada especie, éstas se distribuyeron en tres grupos de 45 plántulas y a cada grupo se le asignó un tratamiento; es decir, cada tratamiento consistió de 45 plántulas, las cuales se cambiaron de posición cada 15 días a partir del inicio del experimento. 
Los tratamientos fueron: (a) sequía (TS), sin riego durante los 120 días del experimento; (b) riego regular (TR), las plántulas se regaron cada $48 \mathrm{hrs}$ y (c) inundado (TI), las plántulas se mantuvieron en condiciones de inundación de manera permanente, manteniendo el nivel del agua a 8-10 $\mathrm{cm}$ sobre el sustrato.

La condición de inundación se logró mediante la sumersión de la maceta de $800 \mathrm{ml}$, con múltiples perforaciones, en el interior de una maceta de $3 \mathrm{I}$. El riego de las plántulas para los tratamientos TR y TI se hizo con agua embotellada. Las plántulas se fertilizaron cada 30 días con $50 \mathrm{ml}$ de solución de Hoagland, formulada para hidroponía (Hoagland y Arnon, 1950), durante los 120 días de duración del experimento (cinco aplicaciones).

\section{Variables medidas}

Las variables de respuesta se midieron a los $0,30,60,90$ y 120 días. La supervivencia se evaluó mediante la identificación de plántulas muertas, consideradas así cuando perdían turgencia en la yema apical, cambiaba la coloración característica de sus hojas y presentaban signos de marchitez. Para evaluar el desarrollo de las plántulas se midió, con una regla graduada en milímetros, la longitud del tallo desde la base de la planta (cuello de la raíz) hasta el ápice del tallo, y se contó el número de hojas completamente expandidas (maduras).

Al final del experimento (120 días) se cosecharon todas las plántulas que sobrevivieron y se midió, empleando una regla graduada en milímetros, la longitud de la raíz principal desde su parte distal más delgada hasta la base de la planta.

La biomasa se calculó secando, en una estufa de convección (Memmert, WISCONSIN, EUA) a $70^{\circ} \mathrm{C}$ durante 72 horas (Cornelissen et al., 2003) de manera separada las hojas, tallo y raíces de cada planta, y con ello se obtuvo la biomasa total, aérea (hojas y tallos) y subterránea (raíz). El peso se registró empleando una balanza analítica digital (0.001 gr, Ohaus, Ohaus, EUA). La proporción raíz:tallo se obtuvo para cada planta como biomasa (g) de raíz entre la biomasa del tallo, y se utiliza para estimar la distribución de biomasa entre la raíz y el tallo (Wilson, 1988).

\section{Análisis estadístico}

El análisis de la respuesta del largo del tallo y el número de hojas de las especies a los tratamientos (TS, TR y TI), se realizó con un modelo lineal mixto de medidas repetidas en el tiempo. Debido a la naturaleza longitudinal de los datos evaluados, se empleó una matriz auto-regresiva de primer orden (Verbeke y Molenberghs, 2009). Los tratamientos se consideraron como factor fijo y el tiempo como factor aleatorio. Se empleó la suma de cuadrados Tipo III, debido a que durante el experimento el diseño se desbalanceó (Fabián et al., 2018) por la mortalidad de las plántulas. Los valores de significancia para las comparaciones pareadas (por ejemplo: entre tratamientos o entre tiempos) se obtuvieron con base en la corrección de Bonferroni ( $p<0.05)$; lo anterior se realizó con la función Imer de la biblioteca Ime4 (Bates et al., 2015) en el programa R 3.24 (R Code Team, 2018).

Al inicio del experimento se empleó el análisis de varianza con un criterio de clasificación para evaluar si había diferencias en la altura y número de hojas de las plántulas. A partir de los 30 días de iniciado el estudio, se utilizó la prueba no paramétrica de $U$ de Mann-Whitney para determinar diferencias $(p<0.05)$ en la altura y número de hojas. Al final del experimento, se evaluaron las diferencias en el largo de raíz y biomasa acumulada $(p<0.05)$ con el mismo estadístico.

\section{Resultados}

\section{Supervivencia}

La supervivencia de las plántulas de $B$. alicastrum en el TI disminuyó 93\% (42 plantas) a partir de los primeros 30 días y $11 \%$ (5 plantas) a los 120 . En el tratamiento TS la supervivencia registrada fue de $89 \%$ (40 plantas) y en TR, de $84 \%$ (38 plantas). Por su parte, la supervivencia de P. sartorianum no se afectó en el tiempo en ningún tratamiento, ya que a los 120 días tuvo 93\% de supervivencia en TS y TR, y 95\% en TI (Fig. 1).

\section{Longitud del tallo}

Al inicio del experimento la longitud del tallo de las plántulas de $B$. alicastrum no presentaron diferencias significativas ( $p>0.05)$ entre tratamientos. Sin embargo, la condición TI limitó el desarrollo de las plántulas a los $30(p<0.05)$ y 60 días $(p<0.05)$ de iniciado el experimento. La longitud del tallo a lo largo del experimento difirió entre los tratamientos TS y TI y entre TR y TI ( $p<0.05)$. Además, se encontraron diferencias significativas $(p<0.0001)$ en la longitud del tallo 

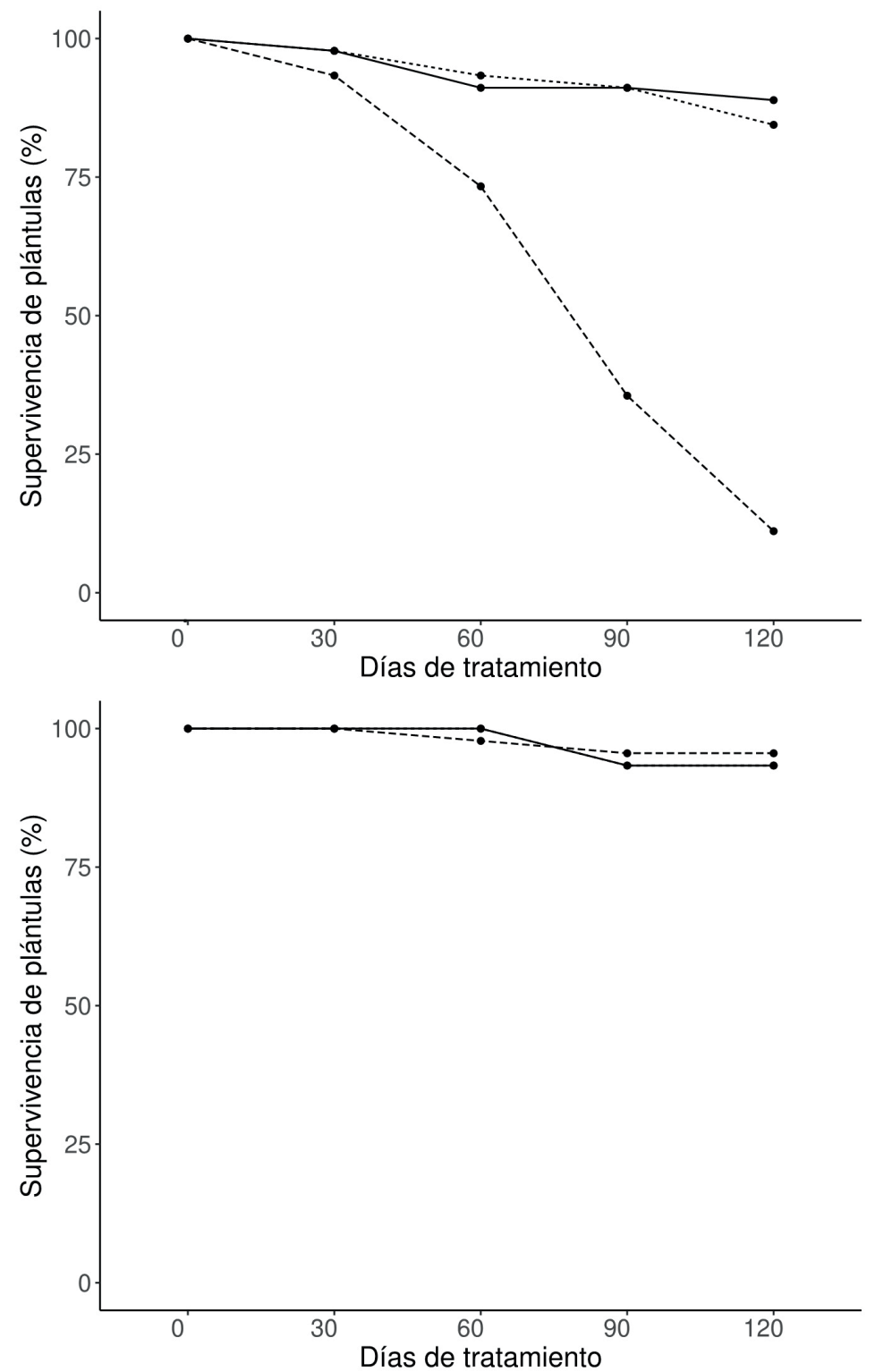
Cuadro 1: Longitud del tallo para la especie Brosimum alicastrum Sw., a los 0, 30, 60, 90 y 120 días del experimento. Los valores son la media ( \pm 1 E.E.) de una $n$ variable para cada periodo. Las letras minúsculas señalan diferencias significativas $(p<0.05)$ entre tratamientos de cada periodo (ANOVA: tiempo 0; Mann-Whitney, U-test: tiempos 30, 60, 90 y 120), las letras mayúsculas señalan las diferencias significativas (Mann-Whitney, U-test p <0.05) en la comparación entre el inicio (0 días) y el final del experimento (120 días) de cada tratamiento.

\begin{tabular}{lccccc}
\hline Tratamiento & 0 días & 30 días & 60 días & 90 días & 120 días \\
\hline Inundado (TI) & $14.89(0.76) \mathrm{aA}$ & $15.16(0.75) \mathrm{b}$ & $16.19(0.74) \mathrm{b}$ & $20.84(0.92) \mathrm{a}$ & $23.50(2.01) \mathrm{aB}$ \\
Sequía (TS) & $15.94(0.56) \mathrm{aA}$ & $17.55(0.53) \mathrm{a}$ & $20.57(0.57) \mathrm{a}$ & $22.45(0.60) \mathrm{a}$ & $26.35(0.63) \mathrm{aB}$ \\
Riego regular (TR) & $16.14(0.56) \mathrm{aA}$ & $17.56(0.59) \mathrm{a}$ & $18.96(0.66) \mathrm{a}$ & $21.47(0.74) \mathrm{a}$ & $26.68(0.63) \mathrm{aB}$ \\
\hline
\end{tabular}

Cuadro 2: Longitud del tallo para la especie Psidium sartorianum (O. Berg) Nied., a los 0, 30, 60, 90 y 120 días del experimento. Los valores son la media ( \pm 1 E.E.) de una $n$ variable para cada periodo. Las letras minúsculas señalan diferencias significativas $(p<0.05)$ entre tratamientos de cada periodo (ANOVA: tiempo 0; Mann-Whitney, U-test: tiempos 30, 60, 90 y 120), las letras mayúsculas señalan las diferencias significativas (MannWhitney, U-test $\mathrm{p}<0.05$ ) en la comparación entre el inicio ( 0 días) y el final del experimento (120 días) de cada tratamiento.

\begin{tabular}{lccccc}
\hline Tratamiento & 0 días & 30 días & 60 días & 90 días & 120 días \\
\hline Inundado (TI) & $15.52(0.65) a A$ & $15.86(0.67) a$ & $16.67(0.76) \mathrm{a}$ & $17.66(0.74) \mathrm{a}$ & $18.69(0.76) \mathrm{aB}$ \\
Sequía (TS) & $13.53(0.74) \mathrm{aA}$ & $14.50(0.79) \mathrm{a}$ & $15.26(0.81) \mathrm{a}$ & $16.07(0.84) \mathrm{a}$ & $18.34(0.86) \mathrm{aB}$ \\
Riego regular (TR) & $14.16(0.68) \mathrm{aA}$ & $14.94(0.67) \mathrm{a}$ & $15.57(0.69) \mathrm{a}$ & $15.97(0.73) \mathrm{a}$ & $18.03(0.73) \mathrm{aB}$ \\
\hline
\end{tabular}

asignadas a los tratamientos. A partir de los 30 días de iniciado el experimento, el tratamiento TI limitó el incremento en el número de hojas en las plántulas de B. alicastrum (Cuadro 3) y P. sartorianum (Cuadro 4). En ambas especies se encontraron diferencias entre los tratamientos a lo largo del tiempo; para B. alicastrum las comparaciones entre los tratamientos TS-TI y TR-TI, fueron diferentes ( $p<0.0001)$; mientras que en $P$. sartorianum la comparación entre los tratamientos TR-TI fue significativa $(p<0.05)$. De igual manera, se registraron diferencias en el número de hojas entre el inicio (0 días) y el final (120 días) del experimento en los tratamientos en ambas especies. Brosimum alicastrum difirió en condiciones de sequía $(p<0.0001)$ y riego regular $(p<0.0001)$, mientras que $P$. sartorianum difiere en condiciones de riego regular $(p<0.05)$.

Al finalizar el experimento las plántulas de las dos especies mostraron el mayor incremento promedio en el número de hojas en TR ( $B$. alicastrum=3.21; $P$. sartorianum=3.93) y en TI se registró una disminución en el número de éstas ( $B$. alicastrum=-0.24; P. sartorianum=-0.68; Cuadro 3 y Cuadro 4).

\section{Longitud de raíz}

La longitud de las raíces de las plántulas de $B$. alicastrum se redujo en las condiciones de los tratamientos TR y TI, mientras que en $P$. sartorianum la longitud de la raíz solamente se afectó negativamente cuando las plántulas permanecieron inundadas permanentemente. La mayor longitud promedio de la raíz se registró a los 120 días en el tratamiento TS, tanto en las plántulas de $B$. alicastrum como en $P$. sartorianum (Fig. 2).

\section{Biomasa (total, aérea y biomasa subterránea)}

La biomasa acumulada en hojas, raíces y total en las plántulas de las dos especies resultó menor en la condición de inundación al finalizar el experimento (120 días). Asimismo, el tratamiento TI tuvo un efecto adverso en la acumulación de la biomasa aérea y de los tallos en $B$. alicastrum (Fig. 3). A su vez, el efecto de la inundación provocó una disminución en la relación raíz/tallo (R/T) en B. alicastrum. La relación $\mathrm{R} / \mathrm{T}$ media en las plántulas de $B$. alicastrum en TI fue menor $(0.36 \pm 0.17)$ respecto de TS $(0.60 \pm 0.21)$ y TR (0.72 \pm 0.52$)$.

\section{Discusión}

En este estudio se realizó un experimento empleando plántulas de Brosimum alicastrum y Psidium sartorianum especies típicas de ambientes contrastantes con el objeto de probar que en los bajos inundables el filtro ambiental 
Cuadro 3: Número de hojas para la especie Brosimum alicastrum Sw., a los 0, 30, 60, 90 y 120 días del experimento. Los valores son la media ( \pm 1 E.E.) de una $n$ variable para cada periodo. Las letras minúsculas señalan diferencias significativas (Mann-Whitney, U-test, $p<0.05$ ) entre tratamientos de cada periodo y las letras mayúsculas señalan las diferencias significativas (Mann-Whitney, U-test $p<0.05$ ) en la comparación entre el inicio (0 días) y el final del experimento (120 días) de cada tratamiento.

\begin{tabular}{lccccc}
\hline Tratamiento & $\mathbf{0}$ días & $\mathbf{3 0}$ días & $\mathbf{6 0}$ días & $\mathbf{9 0}$ días & 120 días \\
\hline Inundado (TI) & $2.64(0.10) \mathrm{aA}$ & $2.57(0.11) \mathrm{a}$ & $2.56(0.13) \mathrm{b}$ & $2.00(0.14) \mathrm{b}$ & $2.40(0.40) \mathrm{aA}$ \\
Sequía (TS) & $2.62(0.08) \mathrm{aA}$ & $2.57(0.22) \mathrm{a}$ & $4.17(0.23) \mathrm{a}$ & $6.00(0.25) \mathrm{a}$ & $5.28(0.23) \mathrm{bB}$ \\
Riego regular (TR) & $2.58(0.09) \mathrm{aA}$ & $2.83(0.19) \mathrm{a}$ & $3.62(0.21) \mathrm{a}$ & $5.44(0.38) \mathrm{a}$ & $5.79(0.34) \mathrm{bB}$ \\
\hline
\end{tabular}

Cuadro 4: Número de hojas para la especie Psidium sartorianum (O. Berg) Nied., a los 0, 30, 60, 90 y 120 días del experimento. Los valores son la media ( \pm 1 E.E.) de una $n$ variable para cada periodo. Las letras minúsculas señalan diferencias significativas (Mann-Whitney, $U$-test, $p<0.05)$ entre tratamientos de cada periodo y las letras mayúsculas señalan las diferencias significativas (Mann-Whitney, U-test $p<0.05$ ) en la comparación entre el inicio (0 días) y el final del experimento (120 días) de cada tratamiento.

\begin{tabular}{llllll}
\hline Tratamiento & 0 días & 30 días & $\mathbf{6 0}$ días & $\mathbf{9 0 ~ d i ́ a s ~}$ & $\mathbf{1 2 0}$ días \\
\hline Inundado (TI) & $10.33(0.73) \mathrm{aA}$ & $9.97(0.70) \mathrm{b}$ & $11.00(0.73) \mathrm{b}$ & $11.36(0.85) \mathrm{b}$ & $9.65(0.83) \mathrm{bA}$ \\
Sequía (TS) & $11.35(0.73) \mathrm{aA}$ & $12.20(0.85) \mathrm{a}$ & $14.00(1.00) \mathrm{a}$ & $14.95(1.20) \mathrm{a}$ & $14.89(1.35) \mathrm{aA}$ \\
Riego regular (TR) & $11.64(0.76) \mathrm{aA}$ & $13.66(1.05) \mathrm{a}$ & $14.68(0.99) \mathrm{a}$ & $15.18(1.19) \mathrm{a}$ & $15.58(1.37) \mathrm{aB}$ \\
\hline
\end{tabular}

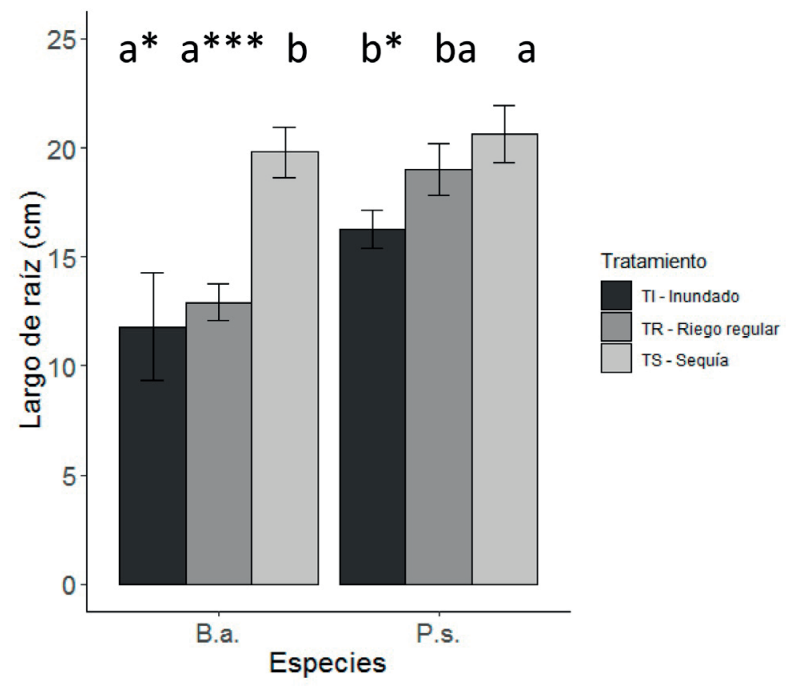

Figura 2: Longitud de la raíz de las plántulas de Brosimum alicastrum Sw. (B.a.) y Psidium sartorianum (O. Berg) Nied. (P.s.) después de 120 días. Para B.a. los valores son la media para TS $n=40$ plantas, TR $n=38$ plantas y en TR $n=5$ plantas. Para P.s. los valores son la media para TS y TR $\mathrm{n}=42$ plantas y en $\mathrm{TI} n=43$ plantas, en ambos casos las barras verticales representan \pm 1 E.E. Letras distintas señalan diferencias significativas entre los tratamientos, $\mathrm{p}<0.05\left({ }^{*}\right), \mathrm{p}<0.01\left({ }^{* *}\right), \mathrm{p}<0.001\left({ }^{* *}\right)$, basada en la prueba de Mann-Whitney (U-test).

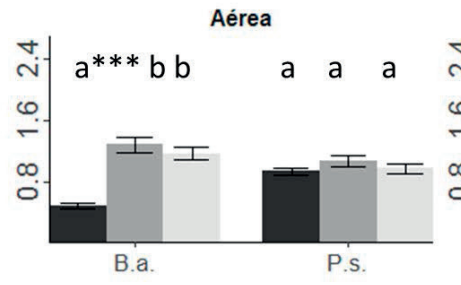

R/T
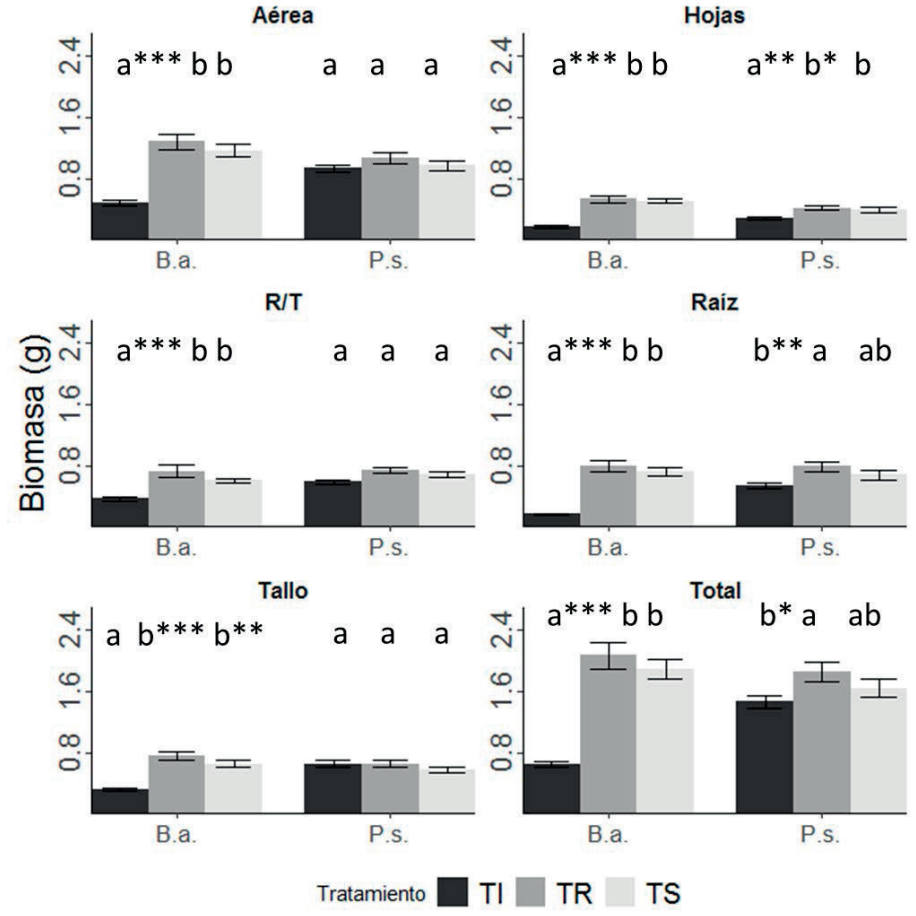

Figura 3: Biomasa promedio aérea, de raíces, hojas, tallo, total y proporción raíz:tallo $(\mathrm{R} / \mathrm{T})$ en las plántulas de Brosimum alicastrum Sw. (B.a.) y Psidium sartorianum (O. Berg) Nied. (P.s.) después de 120 días. Las barras verticales representan \pm 1 E.E. TI: Inundado, TR: Riego regular, TS: Sequía. Letras distintas señalan diferencias significativas entre los tratamientos, $\mathrm{p}<0.05(*), \mathrm{p}<0.01\left({ }^{* *}\right), \mathrm{p}<0.001\left({ }^{* * *}\right)$, basada en la prueba de Mann-Whitney (U-test). 
es determinado por la inundación estacional. Las especies estudiadas son abundantes y representativas de hábitats contrastantes en el área de estudio: se eligieron para probar experimentalmente el efecto del anegamiento, que es un factor abiótico estresante característico de los bajos inundables (Duch-Gary, 1989; Martínez y Galindo-Leal, 2002; Palacio-Aponte et al., 2002) y que puede actuar como un filtro ambiental (Kraft et al., 2015). En diversos estudios se ha evaluado el efecto de la inundación en plántulas de especies arbóreas por un periodo igual o menor a 90 días (López y Kursar, 1999, 2003; Parolin, 2001; Araújo da Paz et al., 2017). Este estudio tuvo una mayor duración (120 días), por lo que cubrió gran parte del tiempo del periodo de inundación reportado en los bajos inundables de la Península de Yucatán (Martínez y Galindo-Leal, 2002; Palacio-Aponte et al., 2002).

El filtro ambiental impuesto por las condiciones de inundación disminuyó la supervivencia de B. alicastrum después de los 30 días de iniciado el experimento; al respecto, existe evidencia de respuestas similares de esta especie en condiciones naturales bajo inundación, originadas ya sea por la cercanía de las plántulas a las márgenes de ríos (Meli et al., 2015) o bien, por la intensidad de las Iluvias producto de huracanes (Bonilla-Moheno y Holl, 2010). Por el contrario, la supervivencia de las plántulas de $P$. sartorianum no se afectó negativamente en ningún tratamiento a lo largo de la duración del experimento. La supervivencia observada en ambas especies en las condiciones experimentales es un reflejo de la acción del filtro ambiental en los bajos inundables, ya que $B$. alicastrum es dominante en selvas no inundables (Pennington y Sarukhán, 2005) mientras que $P$. sartorianum es una especie típica de los bajos inundables (Martínez y Galindo-Leal, 2002; Palacio-Aponte et al., 2002). Otras especies del género Brosimum Sw., como B. lactescens (S. Moore) C.C. Berg. y B. guianense (Aubl.) Huber, al igual que $B$. alicastrum, se desarrollan en las porciones no inundables de los bosques de aguas blancas (várzea en Brasil (Wittmann et al., 2013; Assis et al., 2015). En el caso del género Psidium L., existen varias especies que se distribuyen en los bosques inundables de aguas blancas (várzea) y de aguas negras (igapó). Una de las especies que destacan por su tolerancia al anegamiento es $P$. guajava L., ya que sus plántulas pueden permanecer hasta 90 días en condicio- nes de inundación sin efectos adversos en su supervivencia (Araújo da Paz et al., 2017).

Por otro lado, en condiciones de sequía se ha observado que especies como $P$. acutangulum DC., son afectadas y muestran una alta mortalidad de plántulas, cercana a $70 \%$ (Parolin et al., 2010). No obstante, en este trabajo la especie congenérica estudiada mostró una respuesta opuesta, ya que las plántulas de $P$. sartorianum pudieron sobrevivir al menos 120 días en las dos condiciones experimentales que simulan el filtro ambiental determinado por la sequía e inundación sin que impacten su supervivencia (93\% en TS y TR y 95\% en TI). Dicha respuesta pudo derivar de la adaptación de la especie a la amplia variabilidad ambiental y a la alternancia entre periodos de sequía e inundación como los que se presentan en los bajos inundables de la Península de Yucatán (Martínez y Galindo-Leal, 2002), brindándole la posibilidad de establecerse en áreas sujetas a inundaciones estacionales, lo cual indica que $P$. sartorianum no se ve afectada por el filtro ambiental. Además, las respuestas observadas en este estudio contribuyen a explicar la limitada presencia de $B$. alicastrum en ambientes inundables (Albor-Pinto et al., 2017) y confirman los hallazgos que señalan que la inundación actúa como un filtro ambiental para las especies que se distribuyen en áreas aledañas a los bajos inundables, y cuyas semillas se dispersan al interior de los bajos sin alcanzar su establecimiento y ulterior desarrollo. Lo anterior conduce a diferencias notorias en la composición florística de fragmentos de bosque entre los bajos inundables y las áreas de lomeríos que los circundan (Albor-Pinto et al., 2017).

El filtro ambiental (inundación) limitó el desarrollo del tallo en las especies estudiadas, tal como se ha reportado en otras investigaciones (López y Kursar, 1999, 2003; Parolin, 2001). La misma restricción al crecimiento del tallo se presenta en especies arbóreas que se distribuyen en sabanas inundables; Oliveira et al. (2015) señalan que las especies que habitan ambientes no inundables muestran una mayor susceptibilidad a las condiciones de inundación. Este escenario puede corresponder a la respuesta observada en el experimento con las plántulas de B. alicastrum.

En ambientes estresantes, en los que se presentan inundaciones frecuentes y prolongadas, las plántulas pueden mostrar rápidas elongaciones en el tallo como respuesta 
de escape (Parolin, 2001). Lo anterior no se presentó en las especies estudiadas. Por ejemplo, en las plántulas de $P$. sartorianum la longitud del tallo no tuvo incrementos, pero sobrevivieron a las condiciones de inundación, es decir, sobrevivieron a la acción del filtro ambiental. Por el contrario, se registró que la longitud del tallo fue mayor bajo condiciones de sequía y riego regular, siendo más evidente esta respuesta en $B$. alicastrum con un incremento medio de $10.4 \mathrm{~cm}$. Las plántulas de $P$. sartorianum empleadas en el experimento tuvieron una longitud inicial promedio menor a $20 \mathrm{~cm}$, característica semejante a las plántulas de $P$. acutangulum cuyas tallas no sobrepasan los $50 \mathrm{~cm}$; esta especie ocurre en las porciones inundables de los bosques de aguas blancas (várzea, Parolin et al., 2003). El limitado crecimiento observado en $P$. sartorianum coincide con lo reportado por Wittmann et al. (2013), quienes sugieren que los hábitats inundados por largos periodos se distinguen por incluir especies con bajo crecimiento en altura, tal como ocurre con la especie estudiada y en otras presentes en los bajos inundables de la Península de Yucatán (por ej. Cameraria latifolia L., Coccoloba cozumelensis Hemsl., Croton reflexifolius Kunth, Dalberghia glabra (Mill.) Standl., Eugenia gaumeri Standl., Hyperbaena winzerlingii Standl., Jatropha gaumeri Greenm., Metopium brownei (Jacq.) Urb., Sebastiana adenophora Pax \& K. Hoffm., Terminalia buceras (L.) D.C. Wright, y Terminalia molinetti M. Gómez; (Lundell, 1934; Miranda, 1958; Miranda y Hernández X., 1963; Palacio-Aponte et al., 2002) posiblemente debido a la acción del filtro ambiental, tal como se muestra en este estudio.

Las plantas resistentes a inundaciones mantienen el oxígeno en las raíces, mediante la organogénesis de raíces adventicias (Sauter, 2013) y mediante el transporte de oxígeno para mantener la aireación de las raíces (Graffmann et al., 2008). Otro mecanismo consiste en retener el follaje durante la inundación ante la baja disponibilidad de oxígeno (De Simone et al., 2003). Este último mecanismo podría explicar la supervivencia de $P$. sartorianum ya que las plántulas bajo las condiciones del anegamiento mantuvieron sus hojas durante los 120 días. Por el contrario, en las condiciones que simulan ambientes no inundables registramos un incremento gradual en el número de hojas, el cual inició a los 60 días en B. alicastrum y a los 30 en $P$. sartorianum.
La respuesta de las especies del género Psidium cuando son expuestas a condiciones de inundación varía según la especie; por ejemplo, se ha encontrado que $P$. ovatifolium (O. Berg) Nied., mantiene sus hojas cuando está completamente sumergida, mientras que $P$. acutangulum DC., pierde cerca de $90 \%$ de sus hojas y su caída inicia entre la semana 4 y 16, posterior a la inundación (Wittmann y Parolin, 1999).

En el caso de $B$. alicastrum se encontraron cambios en condiciones de anegamiento tal y como señalan Meli et al. (2015) y López y Kursar (1999; 2003), lo que sugiere que este factor abiótico es determinante en el desarrollo de la especie y permite suponer que su efecto como filtro abiótico es de mayor importancia que el daño causado por la herbivoría de mamíferos (filtro biótico), ya que en estos casos las plántulas tienen la capacidad de producir nuevas hojas (Ballina-Gómez et al., 2008); en este trabajo no se registró recuperación o producción de nuevas hojas en las plántulas expuestas al filtro por inundación.

Respecto del largo de las raíces, López y Kursar (1999; 2003) señalan que la inundación afecta la longitud de la raíz de las especies que habitan en ambientes no inundables $y$, en menor medida, a las especies que ocurren en ambientes inundables. Esto es similar a los resultados de este estudio, ya que la raíz de ambas especies fue afectada por la inundación como filtro ambiental. Por otro lado, la respuesta observada en condiciones de sequía resalta la capacidad de las especies para aprovechar el agua disponible en el subsuelo. Los árboles adultos de B. alicastrum pueden consumir el agua disponible a $2.5 \mathrm{~m}$ de profundidad en el subsuelo (Querateja et al., 2006); capacidad que resulta ventajosa durante la estación seca, permitiendo la adaptación de la especie a las condiciones de sequía u ocupar sitios bien drenados, donde se desarrollan las selvas de las que es un elemento típico (Pennington y Sarukhán, 2005). En este estudio, las plántulas en condiciones de sequía sostuvieron un incremento en la longitud de la raíz, además de la producción de nuevas hojas y crecimiento del tallo, y posiblemente su capacidad de resistir periodos más prolongados de sequía es mucho mayor al periodo de duración de nuestro experimento.

Los resultados sugieren que las respuestas observadas en las especies estudiadas pueden contribuir al diseño de programas de restauración y establecimiento de plan- 
taciones en ambientes estacionalmente inundables, teniendo en consideración el efecto de la alternancia de periodos de sequía-inundación sobre el desarrollo de las plántulas y como un factor abiótico que puede actuar como filtro ambiental. En este sentido, es todavía necesario diseñar estudios que consideren el análisis del efecto sucesivo entre periodos de filtros ambientales determinados por la sequía e inundación temporal, además de estudiar a otras especies características de los bajos para profundizar en la comprensión del efecto del filtrado sobre el conjunto de especies y en consecuencia en la estructuración de los bajos inundables. Además, los escenarios de cambio climático previstos para esta región, señalan una posible reducción en la precipitación (Orellana et al., 2009, Márdero et al., 2014, Estrada-Medina et al., 2016) que podría generar consecuencias negativas directas sobre el mantenimiento de la diversidad y la singularidad florística que distingue a los bajos inundables. Lo anterior refuerza la importancia de contar con un amplio entendimiento de la dinámica del filtro ambiental en ambientes estacionalmente inundables.

\section{Conclusiones}

La inundación afectó severamente la supervivencia de las plántulas Brosimum alicastrum mientras que las plántulas de Psidium sartorianum resultaron tolerantes a las condiciones de inundación y sequía. La condición de anegamiento limitó el crecimiento y acumulación de biomasa en las dos especies estudiadas. Las respuestas observadas contribuyen al entendimiento del efecto del régimen de inundación sobre el establecimiento de las plántulas en los bajos inundables; esto permite comprender la dinámica asociada a la inundación temporal en los bajos y su acción como un filtro ambiental, que explica la ausencia de $B$. alicastrum en dichos ambientes y su presencia en selvas con suelos bien drenados, como en los lomeríos adyacentes a las zonas inundables.

En la actualidad, en la Península de Yucatán se presenta una reducción de la precipitación que puede favorecer el establecimiento y desarrollo de especies no tolerantes a la inundación como podría ser $B$. alicastrum y otras especies de las selvas no inundables, por lo que en futuras investigaciones deberían incluirse otras especies que se localizan en los lomeríos adyacentes a los bajos inundables con objeto de entender los efectos del cambio climático en el establecimiento y regeneración de las selvas de la Península de Yucatán.

\section{Contribución de los autores}

GEMA, AMR, MGE y JAAG concibieron y diseñaron el estudio. GEMA realizó los análisis. GEMA escribió el manuscrito con la ayuda de AMR, MGE, JAAG, y PAMM. Todos los autores contribuyeron a la interpretación de los resultados, la discusión, revisión y aprobación del manuscrito final.

\section{Financiamiento}

Este estudio fue apoyado por El Colegio de la Frontera Sur (Campeche), a través del Fondo de Investigación y DesarroIlo Tecnológico de El Colegio de la Frontera Sur FID-784.

\section{Agradecimientos}

Se agradecen las facilidades brindadas por el Instituto Tecnológico de Chiná, Campeche, México. A Yuriko Pilar Cruz Koizumi, Perla Ortíz Colín, Janeth Chan Morales, Jhoanny del C. Poot Castro, Geovanny J. Canul Hernández, Edwin A. Poot Euan y Carlos F. Vera Mendicuti por el apoyo brindado durante la realización del experimento.

\section{Literatura citada}

Albor-Pinto, C., J., Tun-Garrido y J. J. Ortiz-Díaz. 2017. Diversidad, estructura y afinidades florísticas de un bosque temporalmente inundable de la Península de Yucatán. Revista de Biología Tropical 65(3): 868-880. DOI: https://doi. org/10.15517/rbt.v65i3.26341

Almeida, P. H. R., A. C. Franco, M. T. F. Piedade, V. Vizoni-Scudeller, B. Kruijt y C. S. Ferreira. 2018. Flood tolerance in two tree species that inhabit both the Amazonian floodplain and the dry Cerrado savanna of Brazil. AoBPlants 10(6): 1-15. DOI: https://doi.org/10.1093/aobpla/ply065

Araújo da Paz, A., C. Ribeiro, A. Alves-Azevedo, E. Rodrígues de Lima y F. M. da Silva-Carmo. 2017. Induced flooding as environmental filter for riparian tree species. Environmental and Experimental Botany 139: 31-38. DOI: https://doi. org/10.1016/j.envexpbot.2017.03.017

Assis, R. L., F. Wittmann, M. T. F. Piedade y T. Haugaasen. 2015. Effects of hydroperiod and substrate properties on tree alpha diversity and composition in Amazonian floodplain forests. Plant Ecology 216(1): 41-54. DOI: https://doi.org/10.1007/ s11258-014-0415-y

Ballina-Gómez, H. S., S. Iriarte-Vivar, R. Orellana y L. S. Santiago. 2008. Crecimiento, supervivencia y herbivoría de plántulas 
de Brosimum alicastrum (Moraceae), una especie del sotobosque neotropical. Revista de Biología Tropical 56(4): 2055-2067. DOI: https://doi.org/10.15517/rbt.v5614.5779

Bates, D., M. Mächler, B. M. Bolkery S. y C. Walker. 2015. Fitting linear mixed-effects models using Ime4. Journal of Statistical Software 67(1): 1-48. DOI: https://doi.org/10.18637/jss. v067.i01

Beach, T., S. Luzzadder-Beach, N. Dunning y D. Cook. 2008. Human and natural impacts on fluvial and karst depressions of the Maya Lowlands. Geomorphology 101: 308-331. DOI: https://doi.org/10.1016/j.geomorph.2008.05.019

Beach, T., S. Luzzadder-Beach, N. Dunning y D. Cook. 2016. Climatic changes and collapses in Maya history. Past Global Changes 42(2): 66-67. DOI: https//doi.org/10.22498/pages.24.2.66

Beach, T., N. Dunning, S. Luzzadder-Beach, D. E. Cook y J. Lohse. 2006. Impacts of the ancient Maya on soils and soil erosion in the central Maya Lowlands. Catena 65(2): 166-178. DOI: https://doi.org/10.1016/j.catena.2005.11.007

Beach, T., S. Luzzadder-Beach, D. Cook, S. Krause, C. Doyle, S. Eshleman, G. Wells, N. Dunning, M. L. Brennan, N. Brokaw, M. Cortes-Ricon, G. Hammond, R. Terry, D. Trein y S. Ward. 2018. Stability and instability on Maya Lowlands tropical hillslope soils. Geomorphology 305: 185-208. DOI: https:// doi.org/10.1016/j.geomorph.2017.07.027

Bonilla-Moheno, M. y K. D. Holl. 2010. Direct seeding to restore tropical mature-forest species in areas of slash-and-burn agriculture. Restoration Ecology 18(s2): 438-445. DOI: https:// doi.org/10.1111/j.1526-100X.2009.00580.x

Cadotte, M. W. y C. M. Tucker. 2017. Should environmental filtering be abandoned? Trends in Ecology and Evolution 32(6): 429-437. DOI: https://doi.org/10.1016/j.tree.2017.03.004

Conserva, A., J. L. Campana-Camargo, D. García de Santana y M. T. F. Piedade. 2018. Germinative behavior of ten tree species in white-water floodplain forest in central Amazonia. Folia Geobotanica 53(1): 89-101. DOI: https://doi.org/10.1007/ s12224-017-9284-1

Cornelissen, J. H. C., S. Lavorel, E. Garnier, S. Díaz, N. Buchmann, D. E. Gurvich, P. B. Reich, H. ter Steege, H. D. Morgan, M. G. A. van der Heijden, J. G. Pausas y H. Poorter. 2003. A handbook of protocols for standardised and easy measurement of plant functional traits worldwide. Australian Journal of Botany 51(4): 335-380. DOI: https://doi.org/10.1071/BT02124
De Simone, O., W. J. Junk y W. Schmidt. 2003. Central Amazon floodplain forest: root adaptations to prolonged flooding. Russian Journal of Plant Physiology 50(6): 848-855. DOI: https://doi.org/10.1023/B:RUPP.0000003285.70058.4c

Duch-Gary, J. 1989. Los bajos inundables (ak'alches) de la Península de Yucatán: las expectativas de una evaluación ambiental referida a su aprovechamiento. Universidad Autónoma Chapingo. Chapingo, Estado de México, México. 50 pp.

Dunning, N. P., R. E. Griffin, T. L. Sever, W. A. Saturno y J. G. Jones. 2016. The nature and origins of linear features in the Bajo de Azúcar, Guatemala: Implications for ancient Maya adaptation to a changing environment. Geoarchaeology, An International Journal 32(1): 107-129. DOI: https://doi. org/10.1002/gea.21568

Graffmann, K., W. Grosse, W. J. Junk y P. Parolin. 2008. Pressurized gas transport in Amazonian floodplain trees. Environmental and Experimental Botany 62(3): 371-375. DOI: https://doi. org/10.1016/j.envexpbot.2007.10.013

Estrada-Medina, H., V. Cobos-Gasca, J. L. Acosta-Rodríguez, S. Peña-Fierro, M. Castilla-Martínez, C. Castillo-Carrillo, S. Franco-Brito, D. López-Castillo, M. López-Díaz, W. Luna-Flores, A. Maldonado-Repetto, O. Álvarez-Rivera, J. L. CámaraRomero, A. Morales-Guadarrama, A. M. Moreno-Arjona, B. Pérez-Niño, P. Rodríguez-Lara y R. L. Zapata-Luna. 2016. La sequía de la península de Yucatán. Tecnología y Ciencias del Agua 7(5): 151-165.

Fabián, D., P. Guadarrama, Hernández-Cuevas, L. y J. A. RamosZapata. 2018. Arbuscular mycorrhizal fungi in a coastal wetland in Yucatan, Mexico. Botanical Sciences 96(1): 24-34. DOI: https://dx.doi.org/10.17129/botsci.1216

FLORA DIGITAL. 2019. Flora digital: Península de Yucatán. https:// www.cicy.mx/sitios/flora\%20digital/indice_busqueda.php (consultado marzo de 2019).

Hawes, J. E. y C. A. Peres. 2016. Patterns of plant phenology in Amazonian seasonally flooded and unflooded forest. Biotropica 48(4): 465-475. DOI: https://doi.org/10.1111/ btp. 12315

Hoagland, D. R. y D. I. Arnon. 1950. The water-culture method for growing plants without soil. California Agricultural Experiment Station 347(2): 4-32.

Keddy, P. A. 1992. Assembly and response rules: two goals for predictive community ecology. Journal of Vegetation Science 3(2): 157-164. DOI: https://doi.org/10.2307/3235676 
Kraft, N. J. B., P. B. Adler, O. Godoy, E. C. James, S. Fuller y J. M. Levine. 2015. Community assembly, coexistence and the environmental filtering metaphor. Functional Ecology 29(5): 592-599. DOI: https://doi.org/10.1111/1365-2435.12345

López, O. R. y T. A. Kursar. 1999. Flood tolerance of four tropical tree species. Tree Physiology 19(14): 925-932. DOI: https://doi. org/10.1093/treephys/19.14.925

López, O. R. y T. A. Kursar. 2003. Does flood tolerance explain tree species distribution in tropical seasonally flooded habitats? Ecophysiology 136(2): 193-204. DOI: https://doi.org/10.1007/ s00442-003-1259-7

López, O. R. y T. A. Kursar. 2007. Interannual variation in rainfall, drought stress and seedlings mortality may mediate monodominance in tropical flooded forests. Oecologia 154(1): 3543. DOI: https://doi.org/10.1007/s00442-007-0821-0

Luize, B. G., J. L. L. Magalhães, H. Queiroz, M. A. Lopes, E. M. Venticinque, E. M. Leão de Moraes Novo y T. S. F Silva. 2018. The tree species pool of Amazonian wetland forests: Which species can assemble in periodically waterlogged habitats? PLoS ONE 13(5): e0198130. DOI: https://doi.org/10.1371/journal. pone.0198130

Lundell, C. 1934. Preliminary sketch of the phytogeography of the Yucatan Peninsula. Carnegie Institute of Washington Publications 436: 257-321.

Márdero, S., B. Schmook, Z. Christman, E. Nickl, L. Schneider, J. Rogan y D. Lawrence. 2014. Precipitation variability and adaptation strategies in the Southern Yucatán Peninsula, Mexico: Integrating local knowledge with quantitative analysis. In: Leal Filho, W., F. Alves, S. Caeiro y U. M. Azeiteiro (eds.). International Perspectives on Climate Change: Latin America and beyond. Springer International Publishing. Berlín, Germany. Pp. 189-201.

Martínez, E., y C. Galindo-Leal. 2002. La Vegetación de Calakmul, Campeche, México. Boletín de la Sociedad Botánica de México 71: 7-32. DOI: https://doi.org/10.17129/botsci.1660

Meli, P., J. M. Rey-Benaya, M. Martínez-Ramos y J. Carabias. 2015. Effects of grass clearing and soil tilling on establishment of planted tree seedlings in tropical riparian pastures. New Forests 46(4): 507-525. DOI: https://doi.org/10.1007/s11056015-9479-3

Miranda, F. 1958. Estudios acerca de la vegetación. In: Beltrán, E. (ed.). Los Recursos Naturales del Sureste y su Aprovechamiento. Tomo II. Instituto Mexicano de Recursos Naturales no Renovables. México, D.F., México. Pp. 215-271.
Miranda, F. y E. Hernández X. 1963. Los tipos de la vegetación de México y su clasificación. Boletín de la Sociedad Botánica de México 28: 29-179. DOI: https://doi.org/10.17129/botsci.1084

Myster, R. W. 2015. Flooding $\times$ tree fall gap interactive effects on blackwater forest floristics and physical structure in the Peruvian Amazon. Journal of Plant Interactions 10(1): 126-131.

Oliveira, A. S., C. S. Ferreira, D. Graciano-Ribeiro y A. C. Franco. 2015. Anatomical and morphological modifications in response to flooding by six Cerrado tree species. Acta Botanica Brasilica 29(4): 478-488. DOI: http://doi.org/10.1590/0102$33062014 a b b 0035$

Orellana, R., C. Espadas, C. Conde y C. Gay. 2009. Atlas. Escenarios de cambio climático en la Península de Yucatán. Centro de Investigaciones Científicas de Yucatán-Universidad Nacional Autónoma de México-Consejo Nacional de Ciencia y Tecnología-Secretaría de Desarrollo Urbano y Medio Ambiente del Estado de Yucatán-Gobierno del Estado de Yucatán-Sistema de Investigación, Innovación y Desarrollo Tecnológico del Estado de Yucatán-Organización de las Naciones Unidad-Programa de las Naciones Unidas para el Desarrollo. Mérida, México. 111 pp.

Palacio-Aponte, G. A., R. Noriega-Trejo y P. Zamora-Crescencio. 2002. Caracterización físico-geográfica del paisaje conocido como "bajos inundables". El caso del área natural protegida Balamkín, Campeche. Investigaciones Geográficas 49: 57-73.

Parolin, P. 2001. Seed germination and early establishment of 12 tree species from nutrient-rich and nutrient-poor Central Amazonian floodplains. Aquatic Botany 70(2): 89-103. DOI: https://doi. org/10.1016/S0304-3770(01)00150-4

Parolin, P. 2002. Submergence tolerance vs escape from submergence: two strategies of seedlings establishment in Amazonian floodplains. Environmental and Experimental Botany 48: 177186. DOI: https://doi.org/10.1016/S0098-8472(02)00036-9

Parolin, P., L. V. Ferreira y W. J. Junk. 2003. Germination characteristics and establishment of tree from central Amazonian floodplain. Tropical Ecology 44(2): 157-169.

Parolin, P., C. Lucas, M. T. F. Piedade y F. Wittmann 2010. Drought responses of flood-tolerance trees in Amazonian floodplains. Annals of Botany 105(1): 129-139. DOI: https://doi.org/10.1093/ aob/mcp258

Pennington, T. D. y J. Sarukhán. 2005. Árboles tropicales de México. Manual para la identificación de las principales especies. 3ra ed. Universidad Nacional Autónoma de México y Fondo de Cultura Económica. México, D.F., México. 523 pp. 
Pitman, N. C. A., J. E. Guevara-Andino, M. Austelia, C. E. Cerón, D. A. Neill, W. Palacios, G. Rivas-Torres, M. R. Silman y J. W. Terborgh. 2014. Distribution and abundance of tree species in swamp forests of Amazonian Ecuador. Ecography 37(9): 902-915. DOI: https://doi.org/10.1111/ecog.00774

Querajeta, J. I., H. Estrada-Medina, M. F. Allen, J. Jiménez-Osornio y R. Ruenes. 2006. Utilization of bedrock water by Brosimum alicastrum trees growing on shallow soil atop limestone in dry tropical climate. Plant and Soil 287: 187. DOI: https:// doi.org/10.1007/s11104-006-9065-8

R Core Team. 2018. R: A Language and Environment for Statistical Computing. R Foundation for Statistical Computing. Vienna, Austria. https://www.R-project.org/.

Sánchez, P. E. 1990. Myrtaceae. Flora de Veracruz 62: 1-146.

Sauter, M. 2013. Root responses to flooding. Current Opinion in Plant Biology 16(3): 282-286. DOI: https://doi. org/10.1016/j.pbi.2013.03.013

Schöngart, J., M. T. F. Piedade, S. Ludwingshausen, V. Horna y M. Worbes. 2002. Phenology and stem growth periodicity of tree species in Amazonian floodplain forest. Journal of Tropical Ecology 18(4): 581-597. DOI: https://doi.org/10.1017/ S0266467402002389

Schranm, M. M., A. A. Furtado de Almeida, F. Pinto-Gomes y P. A. Oliveira-Mangabeira. 2005. Effects of soil flooding of leaf gas exchange and growth of two neotropical pioneer tree species. New Forests 29(2): 161-168. DOI: https://doi. org/10.1007/s11056-005-0247-7

Soares, J. F., M. Soares, D. Mugnol, L. Fatarelli, E. F. Santiago, R. M. Mussury y S. P. Quintao-Scalon. 2018. Morphophysiological re- sponses of Ormosia arborea (Fabaceae) seedlings under flooding and post-flooding condition. Australian Journal of Botany 66(7): 489-499. DOI: https://doi.org/10.1071/BT17206

Vega-López, A., J. I. Valdez y V. M. Cetina. 2003. Zonas ecológicas de Brosimum alicastrum Sw. en la costa del Pacífico mexicano. Madera y Bosques 9(1): 25-53. DOI: https://doi. org/10.21829/myb.2003.911287

Verbeke, G. y G. Molenberghs. 2009. Linear mixed models for longitudinal data. Springer. Berlín, Germany. 523 pp.

Webb, C. O. 2000. Exploring the phylogenetic structure of ecological communities: an example for rain forest trees. The American Naturalist 156(2): 145-155. DOI: https://doi. org/10.1086/303378

Wilson, J. B. 1988. A review of evidence on the control of shoot:root ratio in relation to models. Annals of Botany 61(4): 413-449. DOI: https://doi.org/10.1093/oxfordjournals.aob. a087575

Wittmann, F. y P. Parolin. 1999. Phenology of six tree species from Central Amazonian várzea. Ecotropica 5(1): 51-57.

Wittmann, F. y P. Parolin. 2005. Aboveground roots in Amazonian floodplains trees. Biotropica 37(4): 609-619. DOI: https:// doi.org/10.1111/j.1744-7429.2005.00078.x

Wittmann, F., E. Householder, M. T. F. Piedade, R. Lopes de Assis, J. Schöngart, P. Parolin y W. J. Junk. 2013. Habitat specifity, endemism and the neotropical distribution of Amazonian white-water floodplain trees. Ecography 36(6): 690-707. DOI: https://doi.org/10.1111/j.1600-0587.2012.07723.x 